\title{
Excursion Sets of Infinitely Divisble Random Fields with Convolution Equivalent Levy Measure
}

\author{
Rønn-Nielsen, Anders ; Jensen, Eva B. Vedel
}

Document Version

Accepted author manuscript

Published in:

Journal of Applied Probability

DOI:

10.1017/jpr.2017.37

Publication date:

2017

License

Unspecified

Citation for published version (APA):

Rønn-Nielsen, A., \& Jensen, E. B. V. (2017). Excursion Sets of Infinitely Divisble Random Fields with Convolution Equivalent Lévy Measure. Journal of Applied Probability, 54(3), 833-851.

https://doi.org/10.1017/jpr.2017.37

Link to publication in CBS Research Portal

\section{General rights}

Copyright and moral rights for the publications made accessible in the public portal are retained by the authors and/or other copyright owners and it is a condition of accessing publications that users recognise and abide by the legal requirements associated with these rights.

\section{Take down policy}

If you believe that this document breaches copyright please contact us (research.lib@cbs.dk) providing details, and we will remove access to the work immediately and investigate your claim.

Download date: 26. Apr. 2023
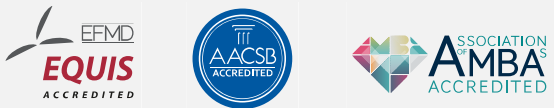


\title{
Excursion Sets of Infinitely Divisble Random Fields with Convolution Equivalent Lévy Measure
}

\author{
Anders Ronn-Nielsen and Eva B. Vedel Jensen
}

Journal article (Accepted version)

CITE: Excursion Sets of Infinitely Divisble Random Fields with Convolution Equivalent Lévy Measure. / Rønn-

Nielsen, Anders ; Jensen, Eva B. Vedel. In: Journal of Applied Probability, Vol. 54, №. 3, २०17, p. 833-851.

This article has been published in a revised form in Journal of Applied Probability.

This version is free to view and download for private research and study only. Not for re-distribution, resale or use in derivative works. [0 Applied Probability Trust २०17.

DOI: 10.1017/jpr.2017.37

Uploaded to Research@CBS: November 2018 


\title{
EXCURSION SETS OF INFINITELY DIVISIBLE RANDOM FIELDS WITH CONVOLUTION EQUIVALENT LÉVY MEASURE
}

\author{
ANDERS RØNN-NIELSEN, ${ }^{*}$ University of Copenhagen
}

EVA B. VEDEL JENSEN, ${ }^{* *}$ Aarhus University

\begin{abstract}
We consider a continuous, infinitely divisible random field in $\mathbb{R}^{d}, d=1,2,3$, given as an integral of a kernel function with respect to a Lévy basis with convolution equivalent Lévy measure. For a large class of such random fields we compute the asymptotic probability that the excursion set at level $x$ contains some rotation of an object with fixed radius as $x \rightarrow \infty$. Our main result is that the asymptotic probability is equivalent to the right tail of the underlying Lévy measure.

Keywords: convolution equivalence; excursion set; infinite divisibility; Lévybased modelling

2010 Mathematics Subject Classification: Primary 60G60

Secondary 60E07;60D05
\end{abstract}

\section{Introduction}

In the present paper we investigate the extremal behaviour of excursion sets for a field $\left(X_{t}\right)_{t \in B}$ defined by

$$
X_{t}=\int_{\mathbb{R}^{d}} f(|t-s|) M(\mathrm{~d} s),
$$

where $M$ is an infinitely divisible, independently scattered random measure on $\mathbb{R}^{d}, f$ is some kernel function, and $B$ is a compact index set. We will assume that the Lévy measure of the random measure $M$ has a convolution equivalent right tail $([5,6,10])$. In [13] it was shown under some regularity conditions that the distribution of $\sup _{t \in B} X_{t}$ has a similar convolution equivalent tail. In the present paper we will be interested in the excursion set

$$
A_{x}=\left\{t: X_{t}>x\right\} .
$$

Under the additional assumption (11) below, we derive the result that the asymptotic probability of the excursion set at level $x$ containing some rotation of an object with a fixed radius $r$ has a tail that is equivalent to the tail of the underlying Lévy measure. A more precise definition of the event that is studied asymptotically is found in Section 2

\footnotetext{
* Postal address: Department of Mathematical Sciences, University of Copenhagen * Email address: arnielsen@math.ku.dk

** Postal address: Department of Mathematics, Aarhus University, and Centre for Stochastic Geometry and Advanced Bioimaging, Aarhus University

** Email address: eva@imf.au.dk
} 
below. Measures with a convolution equivalent tail cover the important cases of an inverse Gaussian and a normal inverse Gaussian (NIG) basis, respectively, see [13].

Lévy models as defined in (1) provide a flexible and tractable modelling framework that recently has been used for a variety of modelling purposes, including modelling of turbulent flows ([4]), growth processes ([8]), Cox point processes ([7]), and brain imaging data ([9]). In [9], a model (1) with $M$ following a NIG distribution was suitable for modelling the neuroscience data under consideration. For such data it is typically of interest to detect for which $t \in B$ a given field obtains values that are significantly large. The results in the present paper will make it possible to discuss whether a cluster of $t \in B$ with large observations jointly form an extreme observation.

For Gaussian random fields it is known that the distribution of the supremum of the field can be approximated by the expected Euler characteristic of an excursion set (see [3] and references therein). The supremum and excursion sets of a non-Gaussian field given by integrals with respect to an infinitely divisible random measure has already been studied, when the random measure has regularly varying tails. Results for the asymptotic distribution of the supremum are found in [11], and these results are refined in [1] and [2], where results are obtained on the asymptotic joint distribution of the number of critical points of the excursion sets. The arguments are - as in the present paper - based on finding the Lévy measure of a dense countable subset of the field. However, the remaining proofs rely heavily on the assumption of regularly varying tails and can therefore not be translated into the convolution equivalent framework.

Note that convolution equivalent distributions have heavier tails than Gaussian distributions and lighter tails than those of regularly varying distributions. The latter statement follows from the fact that convolution equivalent distributions have exponential tails while regularly varying distributions have power function tails.

The present paper is organised as follows. In Section 2 we define the random field (1) and introduce the necessary assumptions. In Section 3 we show three technical lemmas concerning the asymptotic behaviour of deterministic fields. These results will be used in Section 4. In Section 4 we show the main result of the paper. The proof will be in several steps, utilising that $X$ can be decomposed as $X^{1}+X^{2}$, where $X^{1}$ is a compound Poisson sum and $X^{2}$ has lighter tails than $X^{1}$. The proofs in this section will apply techniques that are similar to the proofs in [13].

\section{Preliminaries}

We shall make the same general assumptions as in [13] except for the additional assumption (11) below. For completeness, we will present all assumptions in the following. Consider an independently scattered random measure $M$ on $\mathbb{R}^{d}, d=1,2,3$. Then for a sequence of disjoint sets $\left(A_{n}\right)_{n \in \mathbb{N}} \subseteq \mathbb{R}^{d}$ in $\mathcal{B}\left(\mathbb{R}^{d}\right)$ the random variables $\left(M\left(A_{n}\right)\right)_{n \in \mathbb{N}}$ are independent and satisfy $M\left(\cup A_{n}\right)=\sum M\left(A_{n}\right)$. Assume furthermore that $M(A)$ is infinitely divisible for all $A \in \mathcal{B}\left(\mathbb{R}^{d}\right)$. Then $M$ is called a Lévy basis, see [4] and references therein.

For a random variable $X$ let $C(\lambda \ddagger X)$ denote its cumulant function $\log E\left(e^{i \lambda X}\right)$. We shall assume that the Lévy basis is stationary and isotropic such that for $A \in \mathcal{B}\left(\mathbb{R}^{d}\right)$ the variable $M(A)$ has a Lévy-Khintchine representation given by

$$
C(\lambda \ddagger M(A))=i \lambda a m_{d}(A)-\frac{1}{2} \lambda^{2} \theta m_{d}(A)+\int_{A \times \mathbb{R}}\left(e^{i \lambda z}-1-i \lambda z 1_{[-1,1]}(z)\right) F(\mathrm{~d} s, \mathrm{~d} z),
$$


where $m_{d}$ is the Lebesgue measure on $\left(\mathbb{R}^{d}, \mathcal{B}\left(\mathbb{R}^{d}\right)\right), a \in \mathbb{R}, \theta \geq 0$ and $F$ is a measure on $\mathcal{B}\left(\mathbb{R}^{d} \times \mathbb{R}\right)$ on the form

$$
F(A \times B)=m_{d}(A) \rho(B) .
$$

We assume that $\rho$ has an exponential tail with index $\beta>0$, i.e. for all $y \in \mathbb{R}$

$$
\frac{\rho((x-y, \infty))}{\rho((x, \infty))} \rightarrow e^{\beta y} \quad \text { as } x \rightarrow \infty .
$$

Note that the assumption $\beta>0$ excludes the subexponential case. Let $\rho_{1}$ be a normalization of the restriction of $\rho$ to $(1, \infty)$, and note that $\rho_{1}$ also has an exponential tail with index $\beta>0$. We furthermore assume that

$$
\frac{\left(\rho_{1} * \rho_{1}\right)((x, \infty))}{\rho_{1}((x, \infty))} \rightarrow 2 m \quad \text { as } x \rightarrow \infty,
$$

where $m<\infty$. This makes $\rho_{1}$ a convolution equivalent distribution. (Formally, a distribution is said to be convolution equivalent, if it has an exponential tail and satisfies (5).) Here $\rho_{1} * \rho_{1}$ denotes the convolution. In fact, $m=\int e^{\beta z} \rho_{1}(\mathrm{~d} z)$, cf. [10, Corollary 2.1, (ii)]. Writing $\rho((x, \infty))=L(x) e^{-\beta x}$, it is seen from (4) that for all $y \in \mathbb{R}$

$$
\frac{L(x-y)}{L(x)} \rightarrow 1 \text { as } x \rightarrow \infty .
$$

For each $a, b \in \mathbb{R}$, the limit (6) holds uniformly in $y \in[a, b]$, cf. [10, p. 408]. We furthermore assume

$$
\int z^{2} \rho(\mathrm{d} z)<\infty .
$$

Now assume that $f:[0, \infty) \rightarrow[0, \infty)$ is a strictly decreasing kernel function satisfying

$$
\int_{\mathbb{R}^{d}} f(|s|) \mathrm{d} s<\infty,
$$

and

$$
f(x) \leq \frac{K_{1}}{(x+1)^{d}} \quad \text { for all } x \geq 0
$$

for a finite, positive constant $K_{1}$. Note that (8) follows from (9), when $d=2,3$. Assume furthermore that $f$ is differentiable with $f^{\prime}$ satisfying

$$
\left|f^{\prime}(x)\right| \leq \frac{K_{2}}{(x+1)^{d}} \quad \text { for all } x \geq 0
$$

for a finite, positive constant $K_{2}$. Finally, let $r>0$ be fixed and assume that there exists $g$ such that $f(x) \leq g(x)$ for all $x \geq 0$ and such that

$$
g(x)=f^{\prime}(r)(x-r)+f(r) \text { for all } x \in[0,2 r] .
$$

Note that this in particular is satisfied if $f$ is concave on $[0,2 r]$. We will furthermore choose $g$ on $[2 r, \infty)$ such that it satisfies (8)-(10). 
Let $B$ be a compact, convex subset of $\mathbb{R}^{d}$ with $m_{d}(B)>0$ and define the set $B \oplus C_{r}=\left\{x+y: x \in B, y \in C_{r}(0)\right\}$, where $C_{r}(0)$ is the ball with center in 0 and radius $r$. We consider the family of random variables $\left(X_{t}\right)_{t \in B \oplus C_{r}}$ defined by

$$
X_{t}=\int_{\mathbb{R}^{d}} f(|t-s|) M(\mathrm{~d} s) .
$$

See [13] for existence of the integrals.

Example 2.1. (Gaussian kernel function.) Suppose that $f(x)=e^{-\sigma x^{2}}, \sigma>0$, then the assumptions (8)-(10) are satisfied, and $f$ is concave on the interval $\left[0, \frac{1}{\sqrt{2 \sigma}}\right]$. In particular, the assumption (11) is satisfied for $r \leq \frac{1}{2 \sqrt{2 \sigma}}$.

Example 2.2. (Matérn kernel function.) Suppose that

$$
f(x)=\frac{1}{2^{\eta-1} \Gamma(\eta)}|\lambda x|^{\eta} K_{\eta}(\lambda|x|)
$$

where $K_{\eta}$ is the modified Bessel function of the second kind, index $\eta \geq \frac{1}{2}$, and $\lambda>0$. It can be shown that the Matérn kernel satisfies the assumptions (8)-(10). See [12, Example 2.5] and references therein for details. Furthermore [12, Example 2.5] provides identities for the derivatives of $f$ from which it can be shown that $f$ is concave in an interval $(0, \delta)$ close to 0 , when $\eta>\frac{1}{2}$. In particular, the assumption (11) will be satisfied.

For $s \in B$ let $C_{r}(s)$ be the ball in $\mathbb{R}^{d}$ with radius $r$ and center $s$ and let $S^{d-1}=$ $\left\{\alpha \in \mathbb{R}^{d}:|\alpha|=1\right\}$ be the unit sphere. Let $D \subseteq C_{r}(0)$ be a set with radius $r$ in the sense that there exists $\beta \in S^{d-1}$ such that $\{-r \beta, r \beta\} \subseteq D$. Let furthermore $S O(d)$ denote the special orthogonal group, i.e. the set of all orthogonal matrices with determinant 1. Hence each $R \in S O(d)$ represents a rotation in $\mathbb{R}^{d}$. For $R \in S O(d)$ and $s \in \mathbb{R}^{d}$ we define $D^{R}(s)=R D+s$. Recalling the definition of the excursion set, $A_{x}=\left\{t \in B \oplus C_{r}: X_{t}>x\right\}$, we will be interested in the event

$$
\text { \{there exists } \left.t \in B, R \in S O(d): D^{R}(t) \subseteq A_{x}\right\} \text {. }
$$

Alternatively, this can be expressed as

$$
\left\{\sup _{t_{0} \in B} \sup _{R \in S O(d)} \inf _{t \in D^{R}\left(t_{0}\right)} X_{t}>x\right\} .
$$

Example 2.3. A possible choice of $D$ is $C_{r}(0)$. Here the rotations of $D$ are unnecessary. Another choice could be that $D=\left\{r \alpha_{0},-r \alpha_{0}\right\}$ for a fixed $\alpha_{0} \in S^{d-1}$. A third possibility is the line segment connecting the points $r \alpha$ and $-r \alpha$. For convenience, we let $\alpha_{0}=1, \alpha_{0}=(1,0), \alpha_{0}=(1,0,0)$ for $d=1,2,3$ respectively.

For the study of the extremal behaviour of $\left(X_{t}\right)_{t \in B \oplus C_{r}}$, it will crucial that the field $\left(X_{t}\right)_{t \in T}$ is itself infinitely divisible, with $T=\left(B \oplus C_{r}\right) \cap \mathbb{Q}^{d}$, where $\mathbb{Q}^{d}$ are the rational numbers in $\mathbb{R}^{d}$. For details, see [13] and references therein. The Lévy measure of $\left(X_{t}\right)_{t \in T}$ is the measure $\nu$ on $\left(\mathbb{R}^{T}, \mathcal{B}\left(\mathbb{R}^{T}\right)\right)$ defined by $\nu=F \circ V^{-1}$, where $V: \mathbb{R}^{d} \times \mathbb{R} \rightarrow \mathbb{R}^{T}$ is given by

$$
V(s, z)=(z f(|t-s|))_{t \in T} .
$$


Because of the infinite divisibility of $\left(X_{t}\right)_{t \in T}$, we have the following decomposition, see e.g. [11],

$$
X_{t}=X_{t}^{1}+X_{t}^{2}
$$

where the fields $\left(X_{t}^{1}\right)_{t \in T}$ and $\left(X_{t}^{2}\right)_{t \in T}$ are independent. The first field, $\left(X_{t}^{1}\right)_{t \in T}$, is a compound Poisson sum

$$
X_{t}^{1}=\sum_{n=0}^{N} U_{t}^{n},
$$

where $N$ is Poisson distributed with parameter $\nu(A)<\infty$ and $A=\left\{x \in \mathbb{R}^{T}\right.$ : $\left.\sup _{t \in T} x_{t}>1\right\}$. The fields $\left(U_{t}^{n}\right)_{t \in T}$ are independent and identically distributed with common distribution $\nu_{1}=\nu_{A} / \nu(A)$, where $\nu_{A}$ is the measure on $\left(\mathbb{R}^{T}, \mathcal{B}\left(\mathbb{R}^{T}\right)\right)$ obtained by restricting $\nu$ to $A$. Furthermore $\left(X_{t}^{2}\right)_{t \in T}$ is infinitely divisible with a Lévy measure $\nu_{A^{c}}$, the restriction of $\nu$ to $A^{c}$.

As argued in [13], all the fields $U^{n}, X^{1}$, and $X^{2}$ have continuous extension to $B \oplus C_{r}$. It should furthermore be noted that each of the fields $\left(U_{t}^{n}\right)_{t \in B \oplus C_{r}}$ can be represented by $(Z f(|t-S|))_{t \in B \oplus C_{r}}$, where $(S, Z) \in[0, \infty) \times \mathbb{R}^{d}$ has distribution $F_{1}$, that is the restriction of the measure $F$ to the set

$$
V^{-1}(A)=\left\{(s, z) \in \mathbb{R}^{d} \times \mathbb{R}: \sup _{t \in T} z f(|t-s|)>1\right\} .
$$

\section{Asymptotic results for deterministic fields}

An important property for the arguments in [13] is that for a continuous field $\left(y_{t}\right)_{t \in B \oplus C_{r}}$ it holds for all $s \in B$ that

$$
\inf _{t \in B} \frac{x-y_{t}}{f(|t-s|)}-x+y_{s} \rightarrow 0
$$

as $x \rightarrow \infty$. For the purpose of this paper we shall need a similar but more involved result concerning the asymptotic behaviour of

$$
\inf _{t_{0} \in B} \inf _{R \in S O(d)} \sup _{t \in D^{R}\left(t_{0}\right)} \frac{x-y_{t}}{f(|t-s|)}-\frac{x}{f(r)},
$$

where $S O(d)$ and $D^{R}(t)$ are as defined in the introduction.

Lemma 3.1. Let $\left(y_{t}\right)_{t \in B \oplus C_{r}}$ be a continuous field. Then there exists a function $\lambda_{s}\left(\left(y_{t}\right)_{t \in B \oplus C_{r}}\right)$ such that for each $s \in B$

$$
\inf _{t_{0} \in B} \inf _{R \in S O(d)} \sup _{t \in D^{R}\left(t_{0}\right)} \frac{x-y_{t}}{f(|t-s|)}-\frac{x}{f(r)}+\lambda_{s}\left(\left(y_{t}\right)_{t \in B \oplus C_{r}}\right) \rightarrow 0
$$

as $x \rightarrow \infty$. If $\left(y_{t}\right)_{t \in B \oplus C_{r}}$ is constant-valued and equal to $y$, then $\lambda_{s}\left(\left(y_{t}\right)_{t \in B \oplus C_{r}}\right)=$ $y / f(r)$ for all $s$, and if $y$ is a constant, $\lambda_{s}\left(\left(y+y_{t}\right)_{t \in B \oplus C_{r}}\right)=y / f(r)+\lambda_{s}\left(\left(y_{t}\right)_{t \in B \oplus C_{r}}\right)$.

Furthermore, $\lambda_{s}\left(\left(y_{t}\right)_{t \in B \oplus C_{r}}\right)$ only depends on $\left(y_{t}\right)_{t \in C_{r+\epsilon}(s)}$ for any $\epsilon>0$.

Proof. Let $y^{*}=\sup _{t \in B \oplus C_{r}}$ and $y_{*}=\inf _{t \in B \oplus C_{r}}$. Then the expression in (12) is bounded from above by

$$
\frac{x-y_{*}}{\sup _{t_{0}, R} \inf _{t \in D^{R}\left(t_{0}\right)} f(|t-s|)}-\frac{x}{f(r)}=\frac{x-y_{*}}{f(r)}-\frac{x}{f(r)}=\frac{-y_{*}}{f(r)} .
$$


Similarly, the expression is bounded from below by $-y^{*} / f(r)$. The result for a constant field $\left(y_{t}\right)$ is seen from this, and the result concerning adding a constant to $\left(y_{t}\right)$ follows similarly, when the existence of the limit $\lambda_{s}\left(\left(y_{t}\right)_{t \in B \oplus C_{r}}\right)$ is established. For each $x>0$ we can choose $t_{x} \in B$ and $R_{x} \in S^{d-1}$ such that

$$
\inf _{t_{0} \in B} \inf _{R \in S O(d)} \sup _{t \in D^{R}\left(t_{0}\right)} \frac{x-y_{t}}{f(|t-s|)}-\frac{x}{f(r)}=\sup _{t \in D^{R_{x}}\left(t_{x}\right)} \frac{x-y_{t}}{f(|t-s|)}-\frac{x}{f(r)} .
$$

First, we show that $t_{x} \rightarrow s$. We find

$$
\frac{x-\inf _{t \in C_{r}(s)} y_{t}}{f(r)}=\sup _{t \in C_{r}(s)} \frac{x-y_{t}}{f(r)} \geq \sup _{t \in D^{R_{x}}\left(t_{x}\right)} \frac{x-y_{t}}{f(|t-s|)} \geq \frac{x-y^{*}}{\inf _{t \in D^{R_{x}}\left(t_{x}\right)} f(|t-s|)} .
$$

When using that $\inf _{t \in D^{R_{x}}\left(t_{x}\right)} f(|t-s|) \leq f(r)$ this yields

$$
\frac{x-y^{*}}{x-\inf _{t \in C_{r}(s)} y_{t}} \leq \frac{\inf _{t \in D^{R_{x}}\left(t_{x}\right)} f(|t-s|)}{f(r)} \leq 1
$$

such that $\inf _{t \in D^{R_{x}}\left(t_{x}\right)} f(|t-s|) \rightarrow f(r)$ as $x \rightarrow \infty$. Since furthermore $\inf _{t \in D^{R}\left(t_{0}\right)} f(\mid t-$ $s \mid)<f(r)$ for all $t_{0} \neq s$ and $R \in S O(d)$, we can conclude that $t_{x} \rightarrow s$. From this we can conclude that $\lambda_{s}\left(\left(y_{t}\right)_{t \in B \oplus C_{r}}\right)$ only depends on $y_{t}$ for $t$ close to $C_{r}(s)$.

In fact, we need a stronger version of this result. From differentiability of $f$ in $r$ we have for $u \geq 0$

$$
\frac{1}{f(u)}-\frac{1}{f(r)}=b(u-r)+(u-r) \phi(u-r)
$$

for $b>0$ and some continuous function $\phi$ with $\phi(0)=0$. Using that $f$ is decreasing we find for each $K>0$ that

$$
\begin{aligned}
& x\left(\frac{1}{f(u)}-\frac{1}{f(r)}\right) \leq-b K+\phi(-K / x) \quad \text { for } 0<u<r-\frac{K}{x}, \\
& x\left(\frac{1}{f(u)}-\frac{1}{f(r)}\right) \geq b K+\phi(K / x) \quad \text { for } u>r+\frac{K}{x} .
\end{aligned}
$$

In particular, we can choose $K$ and $x_{0}$ such that for all $x>x_{0}$

$$
\begin{array}{ll}
\frac{x-y_{t}}{f(|t-s|)}-\frac{x}{f(r)}<\frac{-y^{*}}{f(r)} & \text { for }|t-s|<r-\frac{K}{x}, \\
\frac{x-y_{t}}{f(|t-s|)}-\frac{x}{f(r)}>\frac{-y_{*}}{f(r)} & \text { for }|t-s|>r+\frac{K}{x} .
\end{array}
$$

With this choice of $K$ we have for $x>x_{0}$ that

$$
\inf _{t_{0} \in B} \inf _{R \in S O(d)} \sup _{t \in D^{R}\left(t_{0}\right)} \frac{x-y_{t}}{f(|t-s|)}-\frac{x}{f(r)}=\inf _{t_{0} \in B} \inf _{R \in S O(d)} \sup _{t \in D^{R}\left(t_{0}\right) \cap H_{x}} \frac{x-y_{t}}{f(|t-s|)}-\frac{x}{f(r)},
$$

where $H_{x}=\left\{t \in \mathbb{R}^{d}: r-K / x \leq|t-s| \leq r+K / x\right\}$. Define

$$
h(\ell)=\sup \{|\phi(u-r)|: r-\ell \leq u \leq r+\ell\},
$$

and note that $h(\ell) \rightarrow 0$ as $\ell \rightarrow 0$. 
We will show the convergence result by contradiction. To obtain this, we assume that there is a sequence $x_{1}<\tilde{x}_{1}<x_{2}<\tilde{x}_{2}<\ldots$ and constants $a$ and $\epsilon>0$ such that

$$
\sup _{t \in D^{R_{n}}\left(t_{n}\right)} \frac{x_{n}-y_{t}}{f(|t-s|)}-\frac{x_{n}}{f(r)} \leq a \quad \sup _{t \in D^{\tilde{R}_{n}\left(\tilde{t}_{n}\right)}} \frac{\tilde{x}_{n}-y_{t}}{f(|t-s|)}-\frac{\tilde{x}_{n}}{f(r)} \geq a+\epsilon
$$

for all $n$, where $R_{n}=R_{x_{n}}, \tilde{R}_{n}=R_{\tilde{x}_{n}}$ are the corresponding rotation matrices, and $t_{n}=t_{x_{n}}, \tilde{t}_{n}=t_{\tilde{x}_{n}}$ corresponds two the relevant displacements, chosen according to (13). By going to subsequences we can assume that $\left|t_{n}-s\right|$ is decreasing and that $\left(R_{n}\right)$ is convergent. Let $\ell$ be chosen such that $h(\ell)<1 / m$, where $m \in \mathbb{N}$ will be determined later. Let $S_{n}$ be the rotation that is needed to rotate $D^{R_{n+1}}(0)$ into $D^{R_{n}}(0): S_{n} D^{R_{n+1}}(0)=D^{R_{n}}(0)$. Choose $\delta>0$ according to the uniform continuity of $\left(z_{t}\right)_{t \in B \oplus C_{r}}=\left(y_{t} / f(|t-s|)\right)_{t \in B \oplus C_{r}}$ such that $\left|z_{s_{2}}-z_{s_{1}}\right|<\epsilon / 4$ if $\left|s_{2}-s_{1}\right|<\delta$. Furthermore, $\delta$ should be chosen so small that $\delta<\ell / 2$. Choose $\tilde{x}>x_{0}$ such that $\delta+K / \tilde{x}<\ell$. Now choose $n$ such that $\left|t_{n}-t_{n+1}\right|<\delta / 2$, such that $\left|S_{n} u-u\right|<\delta / 2$ for all $u \in B \oplus C_{r}$, and such that $K / x_{n}+\left|t_{n}-t_{n+1}\right|<K / \tilde{x}$.

Recall that $D^{R_{n}}\left(t_{n}\right)$ can be parametrised by $\left\{R_{n} t+t_{n}: t \in D\right\}$ and that similarly, $D^{R_{n+1}}\left(t_{n+1}\right)$ is parametrised by $\left\{R_{n+1} t+t_{n+1}: t \in D\right\}$. Choose $D_{\tilde{x}} \subseteq D$ such that $D^{R_{n}}\left(t_{n}\right) \cap H_{\tilde{x}}=\left\{R_{n} t+t_{n}: t \in D_{\tilde{x}}\right\}$. By the definition of $t_{n}$ we have that

$$
\sup _{t \in D^{R_{n}} \cap H_{\tilde{x}}} \frac{x_{n}-y_{t}}{f(|t-s|)}-\frac{x_{n}}{f(r)} \leq a
$$

Let furthermore $\tilde{D}_{\tilde{x}}^{R_{n+1}}$ be the rotation by $S_{n}$ of $D^{R_{n}}\left(t_{n}\right) \cap H_{\tilde{x}}$ centred in $s: \tilde{D}_{\tilde{x}}^{R_{n+1}}=$ $S_{n}\left(D^{R_{n}}\left(t_{n}\right) \cap H_{\tilde{x}}-s\right)+s$. Now $\tilde{D}_{\tilde{x}}^{R_{n+1}}$ has the form $\left\{R_{n+1} t+\tilde{t}: t \in D_{\tilde{x}}\right\}$ for some $\tilde{t}$; in fact $\tilde{t}=S_{n}\left(t_{n}-s\right)+s$, but that will not be important in the following. Since for $t \in D_{\tilde{x}}$ each $R_{n+1} t+\tilde{t} \in \tilde{D}_{\tilde{x}}^{R_{n+1}}$ is the rotation around $s$ of $R_{n} t+t_{n} \in D^{R_{n}}\left(t_{n}\right) \cap H_{\tilde{x}}$, we have that the distance to $s$ is unchanged. Since furthermore, $\left|R_{n+1} t+\tilde{t}-\left(R_{n} t+t_{n}\right)\right|<\delta$ for $t \in D_{\tilde{x}}$ because of the choice of $S_{n}$, the inequality (17) now leads to

$$
\sup _{t \in \tilde{D}_{\tilde{x}}^{R_{n+1}}} \frac{x_{n}-y_{t}}{f(|t-s|)}-\frac{x_{n}}{f(r)} \leq a+\epsilon / 4
$$

which can be re-parametrised as

$$
\sup _{t \in D_{\tilde{x}}} x_{n}\left(\frac{1}{f\left(\left|R_{n+1} t+\tilde{t}-s\right|\right)}-\frac{1}{f(r)}\right)-z_{R_{n+1} t+\tilde{t}} \leq a+\epsilon / 4 .
$$

Define in the same way $D_{\tilde{x}}^{R_{n+1}}\left(t_{n+1}\right)=\left\{R_{n+1} t+t_{n+1}: t \in D_{\tilde{x}}\right\}$ as a reduced version of $D^{R_{n+1}}\left(t_{n+1}\right)$. By the definition of $t_{n+1}$ we have similarly

$$
\sup _{t \in D_{\tilde{x}}} x_{n}\left(\frac{1}{f\left(\left|R_{n+1} t+t_{n+1}-s\right|\right)}-\frac{1}{f(r)}\right)-z_{R_{n+1} t+t_{n+1}} \leq a,
$$

and by the uniform continuity of $\left(z_{t}\right)$ and the small distance between $t_{n+1}$ and $\tilde{t}$ we have

$$
\sup _{t \in D_{\tilde{x}}} x_{n}\left(\frac{1}{f\left(\left|R_{n+1} t+t_{n+1}-s\right|\right)}-\frac{1}{f(r)}\right)-z_{R_{n+1} t+\tilde{t}} \leq a+\epsilon / 4 .
$$


Note that $D_{\tilde{x}}^{R_{n+1}}\left(t_{n+1}\right)$ is a translation of $\tilde{D}_{\tilde{x}}^{R_{n+1}}$. We shall parametrise all the intermediate translations by

$$
D_{u, \tilde{x}}=\left\{R_{n+1} t+\gamma(u): t \in D_{\tilde{x}}\right\}
$$

for $u \in[0,1]$. Here $\gamma(u)=\tilde{t}+u\left(t_{n+1}-\tilde{t}\right)$ is a linear parametrisation of the line segment from $\tilde{t}$ to $t_{n+1}$. Note that $D_{0, \tilde{x}}=\tilde{D}_{\tilde{x}}^{R_{n+1}}$ and $D_{1, \tilde{x}}=D_{\tilde{x}}^{R_{n+1}}\left(t_{n+1}\right)$. Now define $x(u)=\frac{K}{1-u+C}$ for $u \in[0,1]$, where $C, K>0$ are chosen such that $x(0)=x_{n}$ and $x(1)=x_{n+1}$, see Lemma A.1 in the Appendix. Suppose we can show that

$$
\sup _{t \in D_{\tilde{x}}} x(u)\left(\frac{1}{\left.f\left(\mid R_{n+1} t+\gamma(u)\right)-s \mid\right)}-\frac{1}{f(r)}\right)-z_{R_{n+1} t+\tilde{t}} \leq a+\epsilon / 2
$$

for all $u \in[0,1]$. Then choosing $u$ such that $x(u)=\tilde{x}_{n}$ and defining $\tilde{t}_{n}=\gamma(u)$ gives the inequality

$$
\sup _{t \in D_{\tilde{x}}} \tilde{x}_{n}\left(\frac{1}{\left.f\left(\mid R_{n+1} t+\tilde{t}_{n}\right)-s \mid\right)}-\frac{1}{f(r)}\right)-z_{R_{n+1} t+\tilde{t}} \leq a+\epsilon / 2 .
$$

Using the uniform continuity of $\left(z_{t}\right)$ again together with a reparametrisation gives

$$
\sup _{t \in D_{u, \tilde{x}}} \frac{\tilde{x}_{n}-y_{t}}{f(|t-s|)}-\frac{\tilde{x}_{n}}{f(r)} \leq a+3 \epsilon / 4 .
$$

Note that $D^{R_{n+1}}\left(\tilde{t}_{n}\right) \cap H_{\tilde{x}_{n}} \subseteq D_{u, \tilde{x}}$ due to the choices of $\tilde{x}$ and $x_{n}<\tilde{x}_{n}$. In combination with (15) this gives the desired contradiction to (16).

Thus the proof will be complete, if we can show (20). First, we observe that the cases $u=0$ and $u=1$ follows from (18) and (19). The result for a general $u \in(0,1)$ will follow, if we for any given $t \in D_{\tilde{x}}$ can show that

$$
x(u) F(u) \leq a+\tilde{z}+\epsilon / 2
$$

for all $u \in[0,1]$, where

$$
F(u)=\frac{1}{f(|\tilde{\gamma}(u)-s|)}-\frac{1}{f(r)},
$$

$\tilde{z}=z_{R_{n+1} t+\tilde{t}}$ and $\tilde{\gamma}(u)=R_{n+1} t+\gamma(u)$. For ease of notation, $t$ is suppressed. To obtain this, we will use that for all $t$ such that $r \leq|t-s| \leq r+\ell$ it holds that

$$
(b-1 / m)(|t-s|-r) \leq\left(\frac{1}{f(|t-s|)}-\frac{1}{f(r)}\right) \leq(b+1 / m)(|t-s|-r),
$$

and for $r-\ell \leq|t-s| \leq r$ it holds that

$$
(b+1 / m)(|t-s|-r) \leq\left(\frac{1}{f(|t-s|)}-\frac{1}{f(r)}\right) \leq(b-1 / m)(|t-s|-r),
$$

where we have applied (14) and that $h(\ell)<1 / m$. Note that the assumptions above give that ||$\tilde{\gamma}(u)-s|-r|<\ell$ for all $u \in[0,1]$. Furthermore, note that $F(u)>0$ if and only if $|\tilde{\gamma}(u)-s|-r>0$. We shall consider the cases $(i): F(0), F(1)>0,(i i)$ : $F(0), F(1)<0,($ iii $): F(0)<0, F(1)>0,($ iv $): F(0)>0, F(1)<0$ separately. 
In the case $(i)$ we find using (22) that

$$
(b-1 / m) x(u)(|\tilde{\gamma}(u)-s|-r) \leq a+\tilde{z}+\epsilon / 4
$$

for $u=0,1$. Now let $G(u)$ be the linear interpolation such that $G(0)=(|\tilde{\gamma}(0)-s|-r)$ and $G(1)=(|\tilde{\gamma}(1)-s|-r)$. Then, since $(b-1 / m) x(u) G(u) \leq a+\tilde{z}+\epsilon / 2$ for $u=0,1$, and since $u \mapsto x(u) G(u)$ is monotone, Lemma A.1 in the Appendix gives that the above inequality is satisfied for all $u \in[0,1]$. Since furthermore, $u \mapsto|\tilde{\gamma}(u)-s|$ is seen to be convex, we have that (24) is satisfied for all $u \in[0,1]$. Thus also

$$
(b+1 / m) x(u)(|\tilde{\gamma}(u)-s|-r) \leq(a+\tilde{z}+\epsilon / 4) \frac{b+1 / m}{b-1 / m}
$$

holds for all $u$. Another reference to (22) then gives that

$$
x(u) F(u) \leq(a+\tilde{z}+\epsilon / 4) \frac{b+1 / m}{b-1 / m} .
$$

Now consider the case $(i i)$. Since $F(u)<0$ if both $F(0)<0$ and $F(1)<0$, the property (21) is trivially satisfied, if $a+\tilde{z}+\epsilon / 2 \geq 0$. So assume that $a+\tilde{z}+\epsilon / 2<0$. Then we find similarly using (23) that

$$
x(u) F(u) \leq(a+\tilde{z}+\epsilon / 4) \frac{b-1 / m}{b+1 / m}
$$

The case (iii) is trivially satisfied, since $u \mapsto F(u)$ is increasing. For the case (iv), it is only of interest to show that $x(u) F(u) \leq a+\tilde{z}+\epsilon / 4$ for all $u \in\left[0, u_{0}\right]$, where $F\left(u_{0}\right)=0$. To obtain this, the technique from $(i)$ can be repeated, since here $x(u) F(u) \leq a+\tilde{z}+\epsilon / 4$ for $u=0, u_{0}$.

Now the desired inequality (21) can be obtained from (25) and (26) by letting $m \rightarrow \infty$. Note that this can be done uniformly in $t$, since the field $\left(z_{t}\right)$ is bounded.

The following lemma describes $\lambda_{s}$ for a particularly simple set $D$ :

Lemma 3.2. If $D=\left\{-\alpha_{0} r, \alpha_{0} r\right\}$ with $\alpha_{0}$ as defined in Example 2.3, then

$$
\lambda_{s}\left(\left(y_{t}\right)_{t \in B \oplus C_{r}}\right)=\sup _{\alpha \in S^{d-1}} \frac{1}{2 f(r)}\left(y_{s+\alpha r}+y_{s-\alpha r}\right)
$$

Proof. First, we introduce the notation $D^{\alpha}(s)=\{s-\alpha r, s+\alpha r\}$ for $\alpha \in S^{d-1}$ and $s \in \mathbb{R}^{d}$. Then for $s \in \mathbb{R}^{d}$ fixed we have $\left\{D^{\alpha}(s): \alpha \in S^{d-1}\right\}=\left\{D^{R}(s): R \in S O(d)\right\}$, so for $D$ chosen as in the lemma we can use unit vectors to parametrise all rotations. Now define $u_{s, \alpha}=s+r \alpha$ for $\alpha \in S^{d-1}$ and $u_{s, t, \gamma, \alpha}=s+t \gamma+r \alpha$ for $t \geq 0$ and $\gamma \in S^{d-1}$. The latter parametrises points on the boundary of a ball with radius $r$ and center in $s+t \gamma$. Note that $u_{s, 0, \gamma, \alpha}=u_{s, \alpha}$ and that $\lim _{t \rightarrow 0} u_{s, t, \gamma, \alpha}=u_{s, \gamma, \alpha}$. Furthermore, $\left|u_{t \gamma, \alpha}-s\right|=|t \gamma+r \alpha|=\sqrt{t^{2}+r^{2}+2 t r \cos \angle(\alpha, \gamma)}$, where $\angle(\alpha, \gamma)$ denotes the angle between $\alpha$ and $\gamma$. In the one dimensional case, where $d=1$, we e.g. have $\angle(1,-1)=\pi$. From differentiability of $f$ in $r$ we can write

$$
\begin{aligned}
& \left|\frac{1}{t}\left(\frac{1}{f\left(\left|u_{s, t, \gamma, \alpha}-s\right|\right)}-\frac{1}{f(r)}\right)-\frac{-f^{\prime}(r)}{f(r)^{2}} \cos \angle(\alpha, \gamma)\right| \\
& =\left|\frac{1}{t}\left(\frac{-f^{\prime}(r)}{f(r)^{2}}(|t \gamma+r \alpha|-r)+\phi(|t \gamma+r \alpha|-r)(|t \gamma+r \alpha|-r)\right)-\frac{-f^{\prime}(r)}{f(r)^{2}} \cos \angle(\alpha, \gamma)\right|,
\end{aligned}
$$


where $\phi$ is continuous with $\phi(0)=0$. Using a second order Taylor approximation around 0 of $t \mapsto \sqrt{t^{2}+r^{2}+2 t r \cos \angle(\alpha, \gamma)}$ it is seen that $(|t \gamma+r \alpha|-r) / t$ converges to $\cos \angle(\alpha, \gamma)$ uniformly in $\alpha, \gamma$ as $t \rightarrow 0$. Thus for all $s \in B$

$$
\sup _{\gamma, \alpha}\left|\frac{1}{t}\left(\frac{1}{f\left(\left|u_{s, t, \gamma, \alpha}-s\right|\right)}-\frac{1}{f(r)}\right)-\frac{-f^{\prime}(r)}{f(r)^{2}} \cos \angle(\alpha, \gamma)\right| \rightarrow 0
$$

as $t \rightarrow 0$. Since $y_{u_{s, t, \gamma, \alpha}} \rightarrow y_{u_{s, \alpha}}$ uniformly in $\alpha, \gamma \in S^{d-1}$ due to uniform continuity of the $\left(y_{t}\right)$-field, we find that if $\left(t_{x}\right)$ is a sequence decreasing to 0 such that $x t_{x} \rightarrow C$ as $x \rightarrow \infty$, then

$$
\sup _{\gamma, \alpha}\left|\frac{x-y_{u_{s, t_{x}, \gamma, \alpha}}}{f\left(\left|u_{s, t_{x}, \gamma, \alpha}-s\right|\right)}-\frac{x}{f(r)}-\left(C \frac{-f^{\prime}(r)}{f(r)^{2}} \cos \angle(\alpha, \gamma)-\frac{y_{u_{s, \alpha}}}{f(r)}\right)\right| \rightarrow 0
$$

as $x \rightarrow \infty$. From this we find

$\sup _{\gamma, \alpha}\left|\max _{t \in D^{\alpha}(s+\gamma t)}\left(\frac{x-y_{t}}{f(|t-s|)}-\frac{x}{f(r)}\right)-\max _{t \in D^{\alpha}(s)}\left(C \frac{-f^{\prime}(r)}{f(r)^{2}} \cos \angle(t-s, \gamma)-\frac{y_{t}}{f(r)}\right)\right| \rightarrow 0$

as $x \rightarrow \infty$. Next we claim that for all $\alpha, \gamma \in S^{d-1}$ and $C \geq 0$

$$
\begin{aligned}
& \max _{t \in D^{\alpha}(s)}\left(C \frac{-f^{\prime}(r)}{f(r)^{2}} \cos \angle(t-s, \gamma)-\frac{y_{t}}{f(r)}\right) \\
& =\max \left\{C \frac{-f^{\prime}(r)}{f(r)^{2}} \cos \angle(\alpha, \gamma)-\frac{y_{s+r \alpha}}{f(r)},-C \frac{-f^{\prime}(r)}{f(r)^{2}} \cos \angle(\alpha, \gamma)-\frac{y_{s-r \alpha}}{f(r)}\right\} \\
& \geq \sup _{\alpha \in S^{d-1}} \frac{1}{2 f(r)}\left(y_{s+r \alpha}+y_{s-r \alpha}\right),
\end{aligned}
$$

with equality if

$$
\alpha_{0} \underset{\alpha \in S^{d-1}}{\operatorname{argmax}}\left\{\frac{1}{2 f(r)}\left(y_{s+r \alpha}+y_{s-r \alpha}\right): y_{s+r \alpha} \geq y_{s-r \alpha}\right\},
$$

and furthermore $\gamma_{0}=\alpha_{0}$ and $C_{0}=f(r) /\left(-2 f^{\prime}(r)\right)\left(y_{s+r \alpha}-y_{s-r \alpha}\right)$. For the proposed choice of $\alpha_{0}, \gamma_{0}, C_{0}$ it is easily seen that

$$
C_{0} \frac{-f^{\prime}(r)}{f(r)^{2}} \cos \angle\left(\alpha_{0}, \gamma_{0}\right)-\frac{y_{s+r \alpha_{0}}}{f(r)}=-C_{0} \frac{-f^{\prime}(r)}{f(r)^{2}} \cos \angle\left(\alpha_{0}, \gamma_{0}\right)-\frac{y_{s-r \alpha_{0}}}{f(r)},
$$

and that the common value equals the desired lower bound. It is furthermore seen that any other choice of $\alpha, \gamma, C$ can only increase one of the two terms above.

Now let $\left(\alpha_{n}\right)$ and $\left(\gamma_{n}\right)$ be sequences in $S^{d-1}$, let $\left(t_{n}\right)$ be a sequence of positive numbers, and let $\left(x_{n}\right)$ be a sequence increasing to infinity. Then the results above show that

$$
\liminf _{n \rightarrow \infty} \max _{t \in D_{n}^{\alpha}\left(s+\gamma_{n} t_{n}\right)}\left(\frac{x-y_{t}}{f(|t-s|)}-\frac{x}{f(r)}\right) \geq \sup _{\alpha \in S^{d-1}} \frac{1}{2 f(r)}\left(y_{s+r \alpha}+y_{s-r \alpha}\right)
$$

and that there is equality if $\alpha_{n}=\alpha_{0}, \gamma_{n}=\gamma_{0}$ and $x_{n} t_{n} \rightarrow C_{0}$ with $\alpha_{0}, \gamma_{0}, C_{0}$ as proposed above. Combined with Lemma 3.1 this gives the desired result. 
Lemma 3.3. Let $n \in \mathbb{N}$ and assume for each $i=1, \ldots, n$ that $\left(y_{t}^{i}\right)_{t \in B \oplus C_{r}}$ has the form

$$
y_{t}^{i}=z^{i} f\left(\left|t-s^{i}\right|\right) \quad \text { for all } t \in B \oplus C_{r},
$$

where all $z^{i} \geq 0$ and $s^{i} \in \mathbb{R}^{d}$. Let $g$ be as defined in (11). Define for $s \in \mathbb{R}^{d}$

$$
\varphi(s)=f(r) 1_{B \oplus C_{r}}(s)+1_{\left(B \oplus C_{r}\right)^{c}}(s) \sup _{t \in B} g(|t-s|) .
$$

Then it holds that

$$
\sup _{s \in B} \lambda_{s}\left(\left(\sum_{i=1}^{n} y_{t}^{i}\right)_{t \in B \oplus C_{r}}\right) \leq \frac{1}{f(r)} \sum_{i=1}^{n} z^{i} \varphi\left(s^{i}\right)
$$

and

$$
\sup _{t_{0} \in B} \sup _{\alpha \in S^{d-1}} \inf _{t \in D^{\alpha}\left(t_{0}\right)} \sum_{i=1}^{n} y_{t}^{i} \leq \sum_{i=1}^{n} z^{i} \varphi\left(s^{i}\right) .
$$

Proof. Assume $s^{i} \in B \oplus C_{r}$. For each $\alpha \in S^{d-1}$ and $s \in B$ we find that if $\min \{\mid s+$ $\left.r \alpha-s^{i}|| s-,r \alpha-s^{i} \mid\right\}=r-\delta$ for some $\delta>0$, then $\max \left\{\left|s+r \alpha-s^{i}\right|,\left|s-r \alpha-s^{i}\right|\right\} \geq r+\delta$. Using the assumption (11) then gives

$$
\frac{1}{2}\left(y_{s+r \alpha}^{i}+y_{s-r \alpha}^{i}\right) \leq \frac{z^{i}}{2}(g(r-\delta)+g(r+\delta))=z^{i} f(r)=z^{i} \varphi\left(s^{i}\right) .
$$

This inequality is clearly also satisfied, if both $\left|s+r \alpha-s^{i}\right| \geq r$ and $\left|s-r \alpha-s^{i}\right| \geq r$. If $s^{i} \in\left(B \oplus C_{r}\right)^{c}$ then for all choices of $s \in B$ and $\alpha \in S^{d-1}$ it holds that

$$
\frac{1}{2}\left(y_{s+r \alpha}^{i}+y_{s-r \alpha}^{i}\right) \leq \frac{z^{i}}{2}\left(g\left(\left|s+r \alpha-s^{i}\right|\right)+g\left(\left|s-r \alpha-s^{i}\right|\right)\right) \leq z^{i} \varphi\left(s^{i}\right) .
$$

Recalling that for a given rotation matrix $R \in S O(d)$ there exists $\alpha \in S^{d-1}$ such that $\{s-r \alpha, s+r \alpha\} \subseteq D^{R}(s)$, combined with Lemma 3.2, it is now seen that for each $s \in B$

$$
\begin{aligned}
\lambda_{s}\left(\left(\sum_{i=1}^{n} y_{t}^{i}\right)_{t \in B \oplus C_{r}}\right) & \leq \sup _{\alpha \in S^{d-1}} \frac{1}{2 f(r)}\left(\sum_{i=1}^{n} y_{s+\alpha r}^{i}+\sum_{i=1}^{n} y_{s-\alpha r}^{i}\right) \\
& \leq \sum_{i=1}^{n} \frac{1}{2 f(r)} \sup _{\alpha \in S^{d-1}}\left(y_{s+\alpha r}^{i}+y_{s-\alpha r}^{i}\right) \leq \frac{1}{f(r)} \sum_{i=1}^{n} z^{i} \varphi\left(s^{i}\right) .
\end{aligned}
$$

Taking the supremum over $s \in B$ gives the first statement. For the second statement, we similarly find for each $t_{0} \in B$ and $R \in S O(d)$ that

$$
\begin{aligned}
\inf _{t \in D^{R}\left(t_{0}\right)} \sum_{i=1}^{n} y_{t}^{i} & \leq \min \left\{\sum_{i=1}^{n} y_{t_{0}+r \alpha}^{i}, \sum_{i=1}^{n} y_{t_{0}-r \alpha}^{i}\right\} \\
& \leq \frac{1}{2}\left(\sum_{i=1}^{n} y_{t_{0}+\alpha r}^{i}+\sum_{i=1}^{n} y_{t_{0}-\alpha r}^{i}\right) \leq \sum_{i=1}^{n} z^{i} \varphi\left(s^{i}\right),
\end{aligned}
$$

where, again, $\alpha \in S^{d-1}$ is chosen such that $\{s-r \alpha, s+r \alpha\} \subseteq D^{R}(s)$. The result follows by taking the supremum over $t_{0} \in B$ and $R \in S O(d)$. 


\section{The main theorem}

In this section, we will derive the main result that is Theorem 4.4 below. For $x>0$ we define the following set

$$
\Lambda(x)=\left\{\left(y_{t}\right)_{t \in B \oplus C_{r}}: \sup _{t_{0} \in B} \sup _{R \in S O(d)} \inf _{t \in D^{R}\left(t_{0}\right)} y_{t}>x\right\} .
$$

Note that for a random field $\left(Y_{t}\right)_{t \in B \oplus C_{r}}$ with excursion set $A_{x}=\left\{t \in B \oplus C_{r}: Y_{t}>x\right\}$ we have

$$
P\left(\left(Y_{t}\right)_{t \in B \oplus C_{r}} \in \Lambda(x)\right)=P\left(\text { there exists } t \in B, R \in S O(d): D^{R}(t) \subseteq A_{x}\right) .
$$

The first step will be determining the asymptotic behaviour of excursion sets for a field $U$ with distribution $\nu_{1}$. Recall the definition of $L(x)$ from (6).

Theorem 4.1. Assume that $\left(U_{t}\right)_{t \in B \oplus C_{r}}$ has distribution $\nu_{1}$ and let $\left(y_{t}\right)_{t \in B \oplus C_{r}}$ be continuous. Then

$$
\frac{P\left(\left(U_{t}+y_{t}\right)_{t \in B \oplus C_{r}} \in \Lambda(x)\right)}{L(x / f(r)) \exp (-\beta x / f(r))} \rightarrow \frac{1}{\nu(A)} \int_{B} \exp \left(\beta \lambda_{s}\left(\left(y_{t}\right)_{t \in B \oplus C_{r}}\right)\right) \mathrm{d} s \quad \text { as } x \rightarrow \infty .
$$

Furthermore,

$$
\frac{P\left(\left(U_{t}\right)_{t \in B \oplus C_{r}} \in \Lambda(x)\right)}{L(x / f(r)) \exp (-\beta x / f(r))} \rightarrow \frac{1}{\nu(A)} m_{d}(B) \quad \text { as } x \rightarrow \infty,
$$

and

$$
\frac{P\left(\left(U_{t}+y_{t}\right)_{t \in B \oplus C_{r}} \in \Lambda(x)\right)}{P\left(\left(U_{t}\right)_{t \in B \oplus C_{r}} \in \Lambda(x)\right)} \rightarrow \frac{\int_{B} \exp \left(\beta \lambda_{s}\left(\left(y_{t}\right)_{t \in B \oplus C_{r}}\right)\right) \mathrm{d} s}{m_{d}(B)} \quad \text { as } x \rightarrow \infty \text {. }
$$

Proof. The results (29) and (30) are direct consequences of (28), so we focus on the proof of (28). We can assume that $\left(y_{t}\right)_{t \in B \oplus C_{r}}$ is non-negative: Simply write $x=x^{\prime}-x_{0}$ for a suitable $x_{0}$ such that $\left(x_{0}+y_{t}\right)_{t \in B \oplus C_{r}}$ is non-negative, and find the limit of

$$
\frac{P\left(\left(U_{t}+x_{0}+y_{t}\right)_{t \in B \oplus C_{r}} \in \Lambda\left(x^{\prime}\right)\right)}{L\left(x^{\prime} / f(r)\right) \exp \left(-\beta x^{\prime} / f(r)\right)}
$$

as $x^{\prime} \rightarrow \infty$. We find

$$
\begin{aligned}
P & \left(\left(U_{t}+y_{t}\right)_{t \in B \oplus C_{r}} \in \Lambda(x)\right) \\
= & \frac{1}{\nu(A)} F\left(\left\{(s, z) \in \mathbb{R}^{d} \times \mathbb{R}: \sup _{t_{0} \in B} \sup _{R \in S O(d)} \inf _{t \in D^{R}\left(t_{0}\right)} z f(|t-s|)+y_{t}>x\right\}\right) \\
= & \frac{1}{\nu(A)} F\left(\left\{(s, z) \in \mathbb{R}^{d} \times \mathbb{R}: z>\inf _{t_{0}, R} \sup _{t \in D^{R}\left(t_{0}\right)} \frac{x-y_{t}}{f(|t-s|)}\right\}\right) \\
= & \frac{1}{\nu(A)} \int_{B} L\left(\inf _{t_{0}, \alpha} \sup _{t \in D^{R}\left(t_{0}\right)} \frac{x-y_{t}}{f(|t-s|)}\right) \exp \left(-\beta \inf _{t_{0}, R} \sup _{t \in D^{R}\left(t_{0}\right)} \frac{x-y_{t}}{f(|t-s|)}\right) \mathrm{d} s \\
& +\frac{1}{\nu(A)} \int_{\mathbb{R}^{d} \backslash B} L\left(\inf _{t_{0}, R} \sup _{t \in D^{R}\left(t_{0}\right)} \frac{x-y_{t}}{f(|t-s|)}\right) \exp \left(-\beta \inf _{t_{0}, \alpha} \sup _{t \in D^{R}\left(t_{0}\right)} \frac{x-y_{t}}{f(|t-s|)}\right) \mathrm{d} s .
\end{aligned}
$$


First, we show that the second term in (31) is $o(L(x / f(r)) \exp (-\beta x / f(r)))$. Let $y^{*}=$ $\sup _{s \in B \oplus C_{r}} y_{s}$. We utilise the fact that $L(x) \exp (-\beta x)$ is decreasing, so if $x>y^{*}$, then the second term is

$$
\leq \frac{1}{\nu(A)} \int_{\mathbb{R}^{d} \backslash B} L\left(\frac{x-y^{*}}{f_{0}(s)}\right) \exp \left(-\beta \frac{x-y^{*}}{f_{0}(s)}\right) \mathrm{d} s,
$$

where we have introduced the notation $f_{0}(s)=\sup _{t_{0}, R} \inf _{t \in D^{R}\left(t_{0}\right)} f(|t-s|)$. From the arguments similar to the proof of [13, Theorem 3.1] it can be seen that for all $\gamma>0$ there exists $x_{0}>0$ and $C>0$ such that

$$
\frac{L(a x)}{L(x)} \leq C e^{(a-1) \gamma x} \quad \text { for all } x \geq x_{0}, a \geq 1 .
$$

Note that $f_{0}(s)<f(r)$ for all $s \in \mathbb{R}^{d} \backslash B$ due to convexity of $B$. Combining this with (6), (33) and the fact that $L(x) \exp (-\gamma x) \rightarrow 0$ for all $\gamma>0$, gives that the integrand in (32) is $o(L(x / f(r)) \exp (-\beta x / f(r)))$. If we denote the integrand of $(32)$ by $h(s ; x)$, it follows by the dominated convergence theorem that (32) is $o(L(x / f(r)) \exp (-\beta x / f(r)))$ if we can find an integrable function $g$ such that

$$
\frac{h(s ; x)}{L(x / f(r)) \exp (-\beta x / f(r))} \leq g(s), \quad s \in \mathbb{R}^{d} .
$$

Let $0<\gamma<\beta$. Then, using (33) and the boundedness of $L\left(\left(x-y^{*}\right) / f(r)\right) / L(x / f(r))$, we can find a constant $\tilde{C}$ and $x_{0}>y^{*}$ such that for $x \geq x_{0}$

$\frac{h(s ; x)}{L(x / f(r)) \exp (-\beta x / f(r))} \leq \tilde{C} \exp \left(\beta y^{*} / f(r)\right) \exp \left(-(\beta-\gamma)\left(\frac{1}{f_{0}(s)}-\frac{1}{f(r)}\right)\left(x_{0}-y^{*}\right)\right)$.

Now, choose $D>0$ such that $B \oplus C_{r} \subseteq C_{D}(0)$ and $\sup _{t \in B} f(|t-s|)<f(r)$ for all $s \notin C_{D}(0)$. Then, using (9), we get for $s \notin C_{D}(0)$

$$
f_{0}(s) \leq \sup _{t \in B} f(|t-s|) \leq \sup _{t \in C_{D}(0)} f(|t-s|) \leq \sup _{t \in C_{D}(0)} \frac{1}{(|t-s|+1)^{d}}=\frac{1}{(|s|-D+1)^{d}} .
$$

It follows that the function (34) is integrable.

The theorem now follows by applying dominated convergence to the first term of (31). From Lemma 3.1 we have for $s \in B$

$$
\frac{L\left(\inf _{t_{0}, R} \sup _{t \in D^{R}\left(t_{0}\right)} \frac{x-y_{t}}{f(|t-s|)}\right) \exp \left(-\beta \inf _{t_{0}, R} \sup _{t \in D^{R}\left(t_{0}\right)} \frac{x-y_{t}}{f(|t-s|)}\right)}{L(x / f(r)) \exp (-\beta x / f(r))} \rightarrow e^{\beta \lambda_{s}\left(\left(y_{t}\right)_{t}\right)} .
$$

Using again that $L(x) \exp (-\beta x)$ is decreasing we find for $x$ large

$$
\begin{aligned}
& \left|\frac{L\left(\inf _{t_{0}, R} \sup _{t \in D^{R}\left(t_{0}\right)} \frac{x-y_{t}}{f(|t-s|)}\right) \exp \left(-\beta \inf _{t_{0}, R} \sup _{t \in D^{R}\left(t_{0}\right)} \frac{x-y_{t}}{f(|t-s|)}\right)}{L(x / f(r)) \exp (-\beta x / f(r))}-e^{\beta \lambda_{s}\left(\left(y_{t}\right)_{t}\right)}\right| \\
& \leq \frac{L\left(\left(x-y^{*}\right) / f(r)\right) \exp \left(-\beta\left(x-y^{*}\right) / f(r)\right)}{L(x / f(r)) \exp (-\beta x / f(r))}+e^{\beta \lambda_{s}\left(\left(y_{t}\right)_{t}\right)} \\
& \leq(C+1) e^{\beta y^{*}}
\end{aligned}
$$


where $C$ is chosen such that $L\left(\left(x-y^{*}\right) / f(r)\right) / L(x / f(r)) \leq C$. The result is integrable over $B$.

The next step will be to extend the result of Theorem 4.1 to the case $P\left(\left(U^{1}+\cdots+\right.\right.$ $\left.\left.U^{n}+y_{t}\right)_{t} \in \Lambda(x)\right)$, where $U^{i}, i=1, \ldots, n$, are independent with common distribution $\nu_{1}$. Recall that each $\left(U_{t}^{i}\right)_{t \in B \oplus C_{r}}$ can be represented by $\left(Z^{i} f\left(\left|t-S^{i}\right|\right)\right)_{t \in B \oplus C_{r}}$, where $\left(S^{i}, Z^{i}\right)$ has distribution $F_{1}$. For this purpose we will need the following lemma and corollary.

Lemma 4.1. Let $(S, Z)$ be distributed according to $F_{1}$. Then,

$$
\frac{P(Z \varphi(S)>x)}{L(x / f(r)) \exp (-\beta x / f(r))} \rightarrow \frac{m_{d}\left(B \oplus C_{r}\right)}{\nu(A)} .
$$

In particular, we have

$$
E \exp (\beta / f(r) Z \phi(S))<\infty .
$$

Proof. Similar to the proof of Theorem 4.1 we can write

$$
\begin{aligned}
P(Z \phi(S)>x)= & \frac{1}{\nu(A)} F\left(\left\{(s, z) \in \mathbb{R}^{d} \times \mathbb{R}: z \varphi(s)>x\right\}\right) \\
= & \frac{1}{\nu(A)} \int_{B \oplus C_{r}} L(x / f(r)) \exp (-\beta x / f(r)) \mathrm{d} s \\
& +\frac{1}{\nu(A)} \int_{B \oplus C_{r}} L\left(\frac{x}{\sup _{t \in B} g(|t-s|)}\right) \exp \left(-\beta \frac{x}{\sup _{t \in B} g(|t-s|)}\right) \mathrm{d} s .
\end{aligned}
$$

The first term equals $L(x / f(r)) \exp (-\beta x / f(r))$ times the desired limit. The second term is $o(L(x / f(r)) \exp (-\beta x / f(r))$ by a dominated convergence argument, since $\sup _{t \in B} g(|t-s|)<f(r)$ for all $s \in\left(B \oplus C_{r}\right)^{c}$. The second result follows from [10, Corollary 2.1 (ii)].

Corollary 4.1. Let $U^{1}, U^{2}, \ldots$ be independent and identically distributed with distribution $\nu_{1}$. For all $n \in \mathbb{N}$ it holds that

$$
E \exp \left(\beta \sup _{s \in B} \lambda_{s}\left(\left(U_{t}^{1}+\ldots+U_{t}^{n}\right)_{t \in B \oplus C_{r}}\right)\right)<\infty
$$

Proof. Since each $U^{i}$ has the form $\left(Z^{i} f\left(\left|t-S^{i}\right|\right)\right)_{t \in B \oplus C_{r}}$, the result follows from Lemma 3.3 and Lemma 4.1.

Theorem 4.2. Let $U^{1}, U^{2}, \ldots$ be independent and identically distributed with distribution $\nu_{1}$ and assume that $\left(y_{t}\right)_{t \in B \oplus C_{r}}$ is continuous. For all $n \in \mathbb{N}$ it holds that

$$
\frac{P\left(\left(U_{t}^{1}+\ldots+U_{t}^{n}+y_{t}\right)_{t} \in \Lambda(x)\right)}{P\left(\left(U_{t}^{1}\right)_{t} \in \Lambda(x)\right)} \rightarrow \frac{n}{m_{d}(B)} \int_{B} E e^{\beta \lambda_{s}\left(\left(U_{t}^{1}+\ldots+U_{t}^{n-1}+y_{t}\right)_{t}\right)} \mathrm{d} s
$$

as $x \rightarrow \infty$.

Proof. As in the proof of Theorem 4.1 we can assume that $\left(y_{t}\right)_{t \in B \oplus C_{r}}$ is nonnegative. The result is shown by induction over $n$. For $n=1$, the result is shown in Theorem 4.1. Assume now that the theorem is correct for some $n \in \mathbb{N}$. Let for 
convenience $V=U^{1}+\ldots+U^{n}$ and recall the representation $U_{t}^{i}=Z^{i} f\left(\left|t-S^{i}\right|\right)$. Then we have

$$
\begin{aligned}
& P\left(\left(V_{t}+U_{t}^{n+1}+y_{t}\right)_{t} \in \Lambda(x)\right) \\
& =P\left(\sum_{i=1}^{n} Z^{i} \varphi\left(S^{i}\right)>x / 2, Z^{n+1} \varphi\left(S^{n+1}\right)>x / 2,\left(V_{t}+U_{t}^{n+1}+y_{t}\right)_{t} \in \Lambda(x)\right) \\
& \quad+P\left(\sum_{i=1}^{n} Z^{i} \varphi\left(S^{i}\right) \leq x / 2,\left(V_{t}+U_{t}^{n+1}+y_{t}\right)_{t} \in \Lambda(x)\right) \\
& \quad+P\left(Z^{n+1} \varphi\left(S^{n+1}\right) \leq x / 2,\left(V_{t}+U_{t}^{n+1}+y_{t}\right)_{t} \in \Lambda(x)\right) .
\end{aligned}
$$

The first term is bounded from above by

$$
P\left(\sum_{i=1}^{n} Z^{i} \varphi\left(S^{i}\right)>x / 2\right) P\left(Z^{n+1} \varphi\left(S^{n+1}\right)>x / 2\right) .
$$

In Lemma 4.1 it was shown that the distribution of each $Z^{i} \varphi\left(S^{i}\right)$ is convolution equivalent. Thus both factors are asymptotically equivalent with $\rho_{1}((x /(2 f(r)), \infty))$, and then it follows from the proof of [5, Lemma 2] that the product is $o\left(\left(\rho_{1} * \rho_{1}\right)((x / f(r), \infty))\right)$. In particular, the product above is $o\left(\rho_{1}((x / f(r), \infty))\right)$ due to the convolution equivalence.

The two remaining terms in (35) divided by $P\left(\left(U_{t}^{1}\right)_{t} \in \Lambda(x)\right)$ can be rewritten as follows

$$
\begin{aligned}
& \int_{C_{x}} \frac{P\left(\left(U_{t}^{n+1}+\sum_{i=1}^{n} z^{i} f\left(\left|t-s^{i}\right|\right)+y_{t}\right)_{t} \in \Lambda(x)\right)}{P\left(\left(U_{t}^{1}\right)_{t} \in \Lambda(x)\right)} F_{1}^{* \otimes n}\left(\mathrm{~d}\left(s^{1}, z^{1} ; \ldots ; s^{n}, z^{n}\right)\right) \\
& +\int_{\tilde{C}_{x}} \frac{P\left(\left(V_{t}+z f(|t-s|)+y_{t}\right)_{t} \in \Lambda(x)\right)}{P\left(\left(U_{t}^{1}\right)_{t} \in \Lambda(x)\right)} F_{1}(\mathrm{~d}(s, z)) .
\end{aligned}
$$

Here $F_{1}^{* \otimes n}$ is the $n$-fold product measure of $F_{1}$, and it has been used that $\left(V_{t}\right)_{t}$ can be represented by $\left(\sum_{i=1}^{n} Z^{i} f\left(\left|t-S^{i}\right|\right)\right)_{t}$. The sets $C_{x}$ and $\tilde{C}_{x}$ in (36) are defined by

$$
C_{x}=\left\{\left(s^{1}, z^{1} ; \ldots ; s^{n}, z^{n}\right): \sum_{i=1}^{n} z^{i} \varphi\left(s^{i}\right) \leq x / 2\right\}
$$

and

$$
\tilde{C}_{x}=\{(s, z): z \varphi(s) \leq x / 2\} .
$$

Using Theorem 4.1 and the induction assumption, the two integrands of (36) times $1_{C_{x}}$ and $1_{\tilde{C}_{x}}$ respectively, converge to, as $x \rightarrow \infty$,

$$
f_{1}\left(s^{1}, z^{1} ; \ldots ; s^{n}, z^{n}\right)=\frac{1}{m_{d}(B)} \int_{B} e^{\beta \lambda_{s}\left(\left(y_{t}+\sum_{i=1}^{n} z^{i} f\left(\left|t-s^{i}\right|\right)\right)_{\left.t \in B \oplus C_{r}\right)}\right.} \mathrm{d} s
$$

and

$$
f_{2}(s, z)=\frac{n}{m_{d}(B)} \int_{B} E e^{\beta \lambda_{s}\left(\left(U_{t}^{1}+\ldots+U_{t}^{n-1}+z f(|t-s|)+y_{t}\right)_{t \in B \oplus C_{r}}\right)} \mathrm{d} s,
$$


respectively. We want to show that (36) converges to

$$
\begin{aligned}
& \int f_{1}\left(s^{1}, z^{1} ; \ldots ; s^{n}, z^{n}\right) F_{1}^{* \otimes n}\left(\mathrm{~d}\left(s^{1}, z^{1} ; \ldots ; s^{n}, z^{n}\right)\right)+\int f_{2}(s, z) F_{1}(\mathrm{~d}(s, z)) \\
& =\frac{n+1}{m_{d}(B)} \int_{B} E e^{\beta \lambda_{s}\left(\left(U_{t}^{1}+\ldots+U_{t}^{n}+y_{t}\right)_{t}\right)} \mathrm{d} s .
\end{aligned}
$$

Using Fatou's lemma, it is enough to find integrable functions $g_{1}\left(s^{1}, z^{1} ; \ldots ; s^{n}, z^{n} ; x\right)$ and $g_{2}(s, z ; x)$ that are upper bounds of the two integrands of (36) such that the limits $g_{1}\left(s^{1}, z^{1} ; \ldots ; s^{n}, z^{n}\right)=\lim _{x \rightarrow \infty} g_{1}\left(s^{1}, z^{1} ; \ldots ; s^{n}, z^{n} ; x\right)$ and $g_{2}(s, z)=\lim _{x \rightarrow \infty} g_{2}(s, z ; x)$ exist with

$$
\int_{C_{x}} g_{1}\left(s^{1}, z^{1} ; \ldots ; s^{n}, z^{n} ; x\right) F_{1}^{* \otimes n}\left(\mathrm{~d}\left(s^{1}, z^{1} ; \ldots ; s^{n}, z^{n}\right)\right)+\int_{\tilde{C}_{x}} g_{2}(s, z ; x) F_{1}(\mathrm{~d}(s, z))
$$

converging to the similar integrals with $g_{1}\left(s^{1}, z^{1} ; \ldots ; s^{n}, z^{n}\right)$ and $g_{2}(s, z)$. Using Lemma 3.3 we find that as functions $g_{1}\left(s^{1}, z^{1} ; \ldots ; s^{n}, z^{n} ; x\right)$ and $g_{2}(s, z ; x)$ we can use

$$
g_{1}\left(s^{1}, z^{1} ; \ldots ; s^{n}, z^{n} ; x\right)=\frac{P\left(Z^{1} \varphi\left(S^{1}\right)>x-y^{*}-\sum_{i=1}^{n} z^{i} \varphi\left(s^{i}\right)\right)}{P\left(\left(U_{t}\right)_{t} \in \Gamma(x)\right)},
$$

where as previously $y^{*}=\sup _{t \in B \oplus C_{r}} y_{t}$, and

$$
g_{2}(s, z ; x)=\frac{P\left(\sum_{i=1}^{n} Z^{i} \varphi\left(S^{i}\right)>x-y^{*}-z \varphi(s)\right)}{P\left(\left(U_{t}\right)_{t} \in \Gamma(x)\right)} .
$$

Noting that $P\left(\left(U_{t}\right)_{t} \in \Gamma(x)\right) \sim m_{d}(B) / m_{d}\left(B \oplus C_{r}\right) P\left(Z^{1} \varphi\left(S^{1}\right)>x\right)$ due to Theorem 4.1 and Lemma 4.1, we find that

$g_{1}\left(s^{1}, z^{1} ; \ldots ; s^{n}, z^{n} ; x\right) \rightarrow g_{1}\left(s^{1}, z^{1} ; \ldots ; s^{n}, s^{n}\right)=\frac{m_{d}\left(B \oplus C_{r}\right)}{m_{d}(B)} e^{\beta / f(r)\left(y^{*}+\sum_{k=1}^{n} z^{i} \varphi\left(s^{i}\right)\right)}$,

and since the distribution of $\sum_{i=1}^{n} Z^{i} \varphi\left(S^{i}\right)$ is convolution equivalent, [6, Corollary 2.11] gives

$$
g_{2}(s, z ; x) \rightarrow g_{2}(s, z)=\frac{m_{d}\left(B \oplus C_{r}\right)}{m_{d}(B)} n \cdot e^{\beta / f(r)\left(y^{*}+z \varphi(s)\right)}\left(E e^{\beta / f(r) Z^{1} \varphi\left(S^{1}\right)}\right)^{n-1} .
$$

We observe that

$$
\begin{aligned}
& \int g_{1}\left(s^{1}, z^{1} ; \ldots ; s^{n}, z^{n}\right) F_{1}^{* \otimes n}\left(\mathrm{~d}\left(s^{1}, z^{1} ; \ldots ; s^{n}, z^{n}\right)\right)+\int g_{2}(s, z) F_{1}(\mathrm{~d}(s, z)) \\
& =\frac{m_{d}\left(B \oplus C_{r}\right)}{m_{d}(B)}(n+1) \cdot e^{\beta / f(r) y^{*}}\left(E e^{\beta / f(r) Z^{1} \varphi\left(S^{1}\right)}\right)^{n} .
\end{aligned}
$$

Since the tails of $\sum_{i=1}^{n} Z^{i} \varphi\left(S^{i}\right)$ and $Z^{1} \varphi\left(S^{1}\right)$ in particular are exponential with index $\beta / f(r)$, we have according to [5, Lemma 2$]$ that (37) is asymptotically equal to

$$
e^{\beta / f(r) y^{*}} \frac{P\left(\sum_{i=1}^{n+1} Z^{i} \varphi\left(S^{i}\right)>x\right)}{P\left(Z^{1} \varphi\left(S^{1}\right)>x\right)}
$$

which, by another reference to [6, Corollary 2.11], is seen to converge to (38). 
For a dominated convergence argument, we need the lemma below.

Lemma 4.2. Let $U^{1}, U^{2}, \ldots$ be independent and identically distributed with distribution $\nu_{1}$, and assume that $(S, Z)$ has distribution $F_{1}$. There exists a constant $K$ such that for all $n \in \mathbb{N}$ and all $x \geq 0$

$$
P\left(\left(U_{t}^{1}+\ldots+U_{t}^{n}\right)_{t} \in \Lambda(x)\right) \leq K^{n} P(Z \varphi(S)>x) .
$$

Proof. Since $Z \varphi(S)$ has a convolution equivalent tail according to Corollary 4.1 it follows from [6, Lemma 2.8] that there exists $K$ such that

$$
P\left(\sum_{i=1}^{n} Z^{i} \varphi\left(S^{i}\right)>x\right) \leq K^{n} P(Z \varphi(S)>x) .
$$

The result now follows directly from Lemma 3.3.

Recall that we can write the field $\left(X_{t}\right)_{t \in T}$ as

$$
X_{t}=X_{t}^{1}+X_{t}^{2},
$$

where the field $X^{1}$ is obtained from the fields $U^{1}, U^{2}, \ldots$ and an independent Poisson distributed variable $N$ with parameter $\nu(A)$ by

$$
X_{t}^{1}=\sum_{n=1}^{N} U_{t}^{n}
$$

Theorem 4.3. For each $s \in B$ we have $E \exp \left(\beta \lambda_{s}\left(\left(X_{t}^{1}\right)_{t \in B \oplus C_{r}}\right)\right)<\infty$ and for a continuous field, $\left(y_{t}\right)_{t \in B \oplus C_{r}}$

$$
\lim _{x \rightarrow \infty} \frac{P\left(\left(X_{t}^{1}+y_{t}\right)_{t} \in \Lambda(x)\right)}{L(x / f(r)) \exp (-\beta x / f(r))}=\int_{B} E\left(e^{\beta \lambda_{s}\left(\left(X_{t}^{1}+y_{t}\right)_{t \in B \oplus C_{r}}\right)}\right) \mathrm{d} s .
$$

Proof. The first result follows, since $\lambda_{s}\left(\left(X_{t}^{1}\right)_{t \in B \oplus C_{r}}\right) \leq \frac{1}{f(r)} \sum_{n=0}^{N} Z^{i} \varphi\left(S^{i}\right)$ and $E \exp \left(\beta / f(r) Z^{1} \varphi\left(S^{1}\right)\right)$ is finite. For the proof of the limit result, we use that

$$
P\left(\left(X_{t}^{1}+y_{t}\right)_{t} \in \Lambda(x)\right)=e^{-\nu(A)} \sum_{n=1}^{\infty} \frac{\nu(A)^{n}}{n !} P\left(\left(U_{t}^{1}+\ldots+U_{t}^{n}+y_{t}\right)_{t} \in \Lambda(x)\right) .
$$

Utilising Lemma 4.2 and the notation $y^{*}=\sup _{t \in B \oplus C_{r}} y_{t}$, we find

$$
\begin{aligned}
& \sum_{n=1}^{\infty} \frac{\nu(A)^{n}}{n !} \frac{P\left(\left(U_{t}^{1}+\ldots+U_{t}^{n}+y_{t}\right)_{t} \in \Lambda(x)\right)}{P\left(Z \varphi(S)>x-y^{*}\right)} \\
& \leq \sum_{n=1}^{\infty} \frac{\nu(A)^{n}}{n !} \frac{P\left(\left(U_{t}^{1}+\ldots+U_{t}^{n}\right)_{t} \in \Lambda\left(x-y^{*}\right)\right)}{P\left(Z \varphi(S)>x-y^{*}\right)} \\
& \leq \sum_{n=1}^{\infty} \frac{K^{n} \nu(A)^{n}}{n !} \frac{P\left(Z \varphi(S)>x-y^{*}\right)}{P\left(Z \varphi(S)>x-y^{*}\right)}=\sum_{n=1}^{\infty} \frac{K^{n} \nu(A)^{n}}{n !}<\infty,
\end{aligned}
$$


and furthermore, we obtain from Lemma 4.1 and Theorem 4.2 that

$$
\begin{aligned}
& \lim _{x \rightarrow \infty} \frac{P\left(\left(U_{t}^{1}+\ldots+U_{t}^{n}+y_{t}\right)_{t} \in \Lambda(x)\right)}{P\left(Z \varphi(S)>x-y^{*}\right)} \\
& =\frac{n}{e^{\beta / f(r) y^{*}} m_{d}\left(B \oplus C_{r}\right)} \int_{B} E e^{\beta \lambda_{s}\left(\left(U_{t}^{1}+\ldots+U_{t}^{n-1}+y_{t}\right)_{t}\right)} \mathrm{d} s .
\end{aligned}
$$

with the convention that $U_{t}^{1}+\ldots+U_{t}^{n-1}=0$ if $n=1$. Then, dominated convergence gives

$$
\begin{aligned}
& \lim _{x \rightarrow \infty} \frac{P\left(\left(X_{t}^{1}+y_{t}\right)_{t} \in \Lambda(x)\right)}{P\left(Z \varphi(S)>x-y^{*}\right)} \\
& =\frac{e^{-\nu(A)}}{e^{\beta / f(r) y^{*}} m_{d}\left(B \oplus C_{r}\right)} \sum_{n=1}^{\infty} \frac{\nu(A)^{n}}{n !} n \int_{B} E e^{\beta \lambda_{s}\left(\left(U_{t}^{1}+\ldots+U_{t}^{n-1}+y_{t}\right)_{t}\right)} \mathrm{d} s \\
& \frac{\nu(A)}{e^{\beta / f(r) y^{*}} m_{d}\left(B \oplus C_{r}\right)} \sum_{n=0}^{\infty} e^{-\nu(A)} \frac{\nu(A)^{n}}{n !} \int_{B} E e^{\beta \lambda_{s}\left(\left(U_{t}^{1}+\ldots+U_{t}^{n}+y_{t}\right)_{t}\right)} \mathrm{d} s \\
& =\frac{\nu(A)}{e^{\beta / f(r) y^{*}} m_{d}\left(B \oplus C_{r}\right)} \int_{B} E\left(e^{\beta \lambda_{s}\left(\left(U_{t}^{1}+\ldots+U_{t}^{N}+y_{t}\right)_{t}\right)}\right) \\
& =\frac{\nu(A)}{e^{\beta / f(r) y^{*}} m_{d}\left(B \oplus C_{r}\right)} \int_{B} E\left(e^{\beta \lambda_{s}\left(\left(X_{t}^{1}+y_{t}\right)_{t}\right)}\right)
\end{aligned}
$$

which with a final reference to Theorem 4.1 and Lemma 4.1 concludes the proof.

The theorem below is the main result of our paper. In the formulation of the theorem, we explicitly state the assumptions under which the limit holds.

Theorem 4.4. Under the assumptions (2)-(7) on $M$ and (8)-(11) on $f$, then it holds that $E \exp \left(\beta \lambda_{t_{0}}\left(\left(X_{t}\right)_{t \in B \oplus C_{r}}\right)<\infty\right.$ and

$$
\lim _{x \rightarrow \infty} \frac{P\left(\sup _{t_{0} \in B} \sup _{R \in S O(d)} \inf _{t \in D^{R}\left(t_{0}\right)} X_{t}>x\right)}{L(x / f(r)) \exp (-\beta x / f(r))}=E \exp \left(\beta \lambda_{t_{0}}\left(\left(X_{t}\right)_{t \in B \oplus C_{r}}\right) m_{d}(B)\right.
$$

as $x \rightarrow \infty$ with $t_{0} \in B$ arbitrarily chosen, and where $\lambda_{t_{0}}$ is as defined in Lemma 3.1.

Proof. First we note that $E \exp \left(\gamma \sup _{t \in B \oplus C_{r}} X_{t}^{2}\right)<\infty$ for all $\gamma>0$ according to [13, Lemma 4.1]. Since furthermore

$$
\lambda_{t_{0}}\left(\left(X_{t}\right)_{t}\right) \leq \lambda_{t_{0}}\left(\left(X_{t}^{1}+\sup _{t} X_{t}^{2}\right)_{t}\right)=\lambda_{t_{0}}\left(\left(X_{t}^{1}\right)_{t}\right)+\sup _{t} X_{t}^{2} / f(r)
$$

due to Lemma 3.1, the first statement follows from the first statement in Theorem 4.3. Let $\pi$ be the distribution of $\left(X_{t}^{2}\right)_{t \in B \oplus C_{r}}$. We find that

$$
\frac{P\left(\left(X_{t}\right)_{t} \in \Lambda(x)\right)}{P\left(\left(X_{t}^{1}\right)_{t} \in \Lambda(x)\right)}=\int \frac{P\left(\left(X_{t}^{1}+y_{t}\right)_{t} \in \Lambda(x)\right)}{P\left(\left(X_{t}^{1}\right)_{t} \in \Lambda(x)\right)} \pi(\mathrm{d} y)=\int f(y ; x) \pi(\mathrm{d} y),
$$

with

$$
f(y ; x)=\frac{P\left(\left(X_{t}^{1}+y_{t}\right)_{t} \in \Lambda(x)\right)}{P\left(\left(X_{t}^{1}\right)_{t} \in \Lambda(x)\right)} .
$$


From Theorem 4.3 it is seen that

$$
f(y ; x) \rightarrow f(y)=\frac{\int_{B} E\left(e^{\beta \lambda_{s}\left(\left(X_{t}^{1}+y_{t}\right)_{t \in B \oplus C_{r}}\right)}\right) \mathrm{d} s}{\int_{B} E\left(e^{\beta \lambda_{s}\left(\left(X_{t}^{1}\right)_{t \in B \oplus C_{r}}\right)}\right) \mathrm{d} s} \quad \text { as } x \rightarrow \infty .
$$

If we can show that

$$
\int f(y ; x) \pi(\mathrm{d} y) \rightarrow \int f(y) \pi(\mathrm{d} y)
$$

as $x \rightarrow \infty$, then the theorem follows with another reference to Theorem 4.3 and by recalling that $\left(X_{t}\right)_{t \in B \oplus C_{r}}$ is stationary. According to Fatou's lemma, (39) follows if we can find integrable non-negative functions $g(y ; x)$ and $g(y)$ such that

$$
\begin{aligned}
f(y ; x) & \leq g(y ; x), \\
g(y ; x) & \rightarrow g(y), \\
\int g(y ; x) \pi(\mathrm{d} y) & \rightarrow \int g(y) \pi(\mathrm{d} y) .
\end{aligned}
$$

For this purpose, let

$$
g(y ; x)=\frac{P\left(\left(X_{t}^{1}+\sup _{t} y_{t}\right)_{t} \in \Lambda(x)\right)}{P\left(\left(X_{t}^{1}\right)_{t} \in \Lambda(x)\right)} .
$$

Then, (40) is satisfied. Furthermore, using Theorem 4.3 and Lemma 3.1, we find that (41) is fulfilled with $g(y)=e^{\beta / f(r) \sup _{t} y_{t}}$. To prove (42), we have that

$$
\int g(y ; x) \pi_{2}(\mathrm{~d} y)=\frac{P\left(\sup _{t_{0}, R} \inf _{t \in D^{R}\left(t_{0}\right)} X_{t}^{1}+\sup _{t} X_{t}^{2}>x\right)}{P\left(\sup _{t_{0}, R} \inf _{t \in D^{R}\left(t_{0}\right)} X_{t}^{1}>x\right)} .
$$

Note that $\sup _{t_{0}, R} \inf _{t \in D^{R}\left(t_{0}\right)} X_{t}^{1}$ has a convolution equivalent tail according to Theorem 4.3 and [10, Lemma 2.4 (i)]. Since $E \exp \left(\gamma \sup _{t} X_{t}^{2}\right)<\infty$ for all $\gamma>0$ we have from [10, Lemma 2.1] and [10, Lemma 2.4 (ii)] that

$$
\begin{aligned}
& \lim _{x \rightarrow \infty} \frac{P\left(\sup _{t_{0}, R} \inf _{t \in D^{R}\left(t_{0}\right)} X_{t}^{1}+\sup _{t} X_{t}^{2}>x\right)}{P\left(\sup _{t_{0}, R} \inf _{t \in D^{R}\left(t_{0}\right)} X_{t}^{1}>x\right)} \\
& \quad=E\left(\exp \left(\beta / f(r) \sup _{t} X_{t}^{2}\right)\right)=\int g(y) \pi(\mathrm{d} y) .
\end{aligned}
$$

It follows that (42) is fulfilled.

\section{Appendix A.}

The following simple lemma will be used in Lemma 3.1:

Lemma A.1. Let $0<x_{n}<x_{n+1}$ be given. Then there exists constants $C, D>0$ such that $x:[0,1] \rightarrow[0, \infty)$ defined by

$$
x(u)=\frac{C}{1-u+D}
$$

is strictly increasing with $x(0)=x_{n}$ and $x(1)=x_{n+1}$. Furthermore, if $g(u)=a u+b$, then $u \mapsto x(u) g(u)$ is monotone on $[0,1]$.

Proof. Any function on the form (43) is clearly strictly increasing on $[0,1]$. The constants $C, D$ are found by straightforward manipulations. The last result is obtained by differentiating $u \mapsto x(u) g(u)$. 


\section{Acknowledgements}

This work was supported by Centre for Stochastic Geometry and Advanced Bioimaging, funded by the Villum Foundation.

\section{References}

[1] Adler, R., Samorodnitsky, G. And Taylor, J. E. (2010). Excursion sets of three classes of stable random fields. Adv. Appl. Prob. 42 293-318.

[2] Adler, R., Samorodnitsky, G. And Taylor, J. E. (2013). High level excursion set geometry for non-Gaussian infinitely divisible random fields. Ann. Prob. 41 (1) 134-169.

[3] Adler, R. And Taylor, J. E. (2007). Random Fields and Geometry. Springer, New York.

[4] Barndorff-Nielsen, O. And Schmiegel, J (2004). Lévy based tempo-spatial modelling with applications to turbulence. Uspekhi Mat. Nauk. 159 63-90.

[5] Cline, D. B. H. (1986). Convolution tails, product tails and domains of attraction. Prob. Th. Rel. Fields 72 529-557.

[6] Cline, D. B. H. (1987). Convolutions of distributions with exponential and subexponential tails. J. Austral. Math. Soc. 43 347-365.

[7] Hellmund, G., Prokešová, M and Jensen, E. B. V. (2008). Lévy based Cox point processes. Adv. Appl. Prob. 40 603-629.

[8] Jónsdóttir, K. Ý., Schmiegel, J. And Jensen, E.B.V. (2008). Lévy-based growth models. Bernoulli 14(1) 62-90.

[9] Jónsdóttir, K. Ý., Rønn-Nielsen, A., Mouridsen, K. And Jensen, E.B.V. (2013). Lévy-based modelling in brain imaging. Scand. J. Stat. 40(3) 511-529.

[10] Pakes, A. (2004). Convolution equivalence and infinite divisibility. J. Appl. Prob. 41 407-424.

[11] Rosiński, J. And Samorodnitsky,G. (1993). Distributions of subadditive functionals of sample paths of infinitely divisible processes. Ann. Probab. 21 9961014.

[12] Rønn-Nielsen, A. And Jensen, E.B.V. (2014): Tail asymptotics for the supremum of an infinitely divisible field with convolution equivalent Lévy measure. CSGB Research Report 2014-09.

[13] Rønn-Nielsen, A. And Jensen, E.B.V. (2016): Tail asymptotics for the supremum of an infinitely divisible field with convolution equivalent Lévy measure. J. Appl. Prob. 53 244-261. 